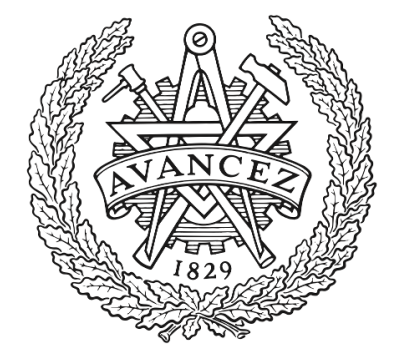

CHALMERS

UNIVERSITY OF TECHNOLOGY

\title{
High power mm-wave spatial power combiner employing on-chip isolation resistors
}

Downloaded from: https://research.chalmers.se, 2023-04-26 03:57 UTC

Citation for the original published paper (version of record):

Roev, A., Maaskant, R., Ivashina, M. et al (2020). High power mm-wave spatial power combiner employing on-chip isolation resistors. 14th European Conference on Antennas and Propagation, EuCAP 2020. http://dx.doi.org/10.23919/EuCAP48036.2020.9135565

N.B. When citing this work, cite the original published paper. 


\title{
High Power mm-Wave Spatial Power Combiner Employing On-Chip Isolation Resistors
}

\author{
Artem Roev*, Rob Maaskant ${ }^{* \dagger}$, Marion K. Matters-Kammerer ${ }^{\dagger}$, Marianna Ivashina*, \\ *Department of Electrical Engineering, Chalmers University of Technology, Gothenburg, Sweden, roev@chalmers.se \\ ${ }^{\dagger}$ Department of Electrical Engineering, Eindhoven University of Technology, Eindhoven, The Netherlands, r.maaskant@tue.nl
}

\begin{abstract}
A spatial power combiner interfacing four power amplifiers (PAs) with isolation load resistors to a single substrate integrated waveguide (SIW) is presented. The isolation load resistors are envisioned on-chip and have been optimized to provide both the optimal active load impedance for the interconnected PAs as well as to mitigate undesired power combiner coupling effects due to non-equal excitations between PA channels. The proposed solution is compared to an ideal Wilkinson combiner in the presence of non-ideal PAs. The main performance targets are the combined output power, gain, and power efficiency at the 1-dB compression point. Simulation results demonstrate that introducing isolation load resistors allows to significantly reduce the impact of a non-uniform excitation on the combiner performance metrics.
\end{abstract}

Index Terms - spatial power combining, mode converter, substrate integrated waveguide, integration, MMICs, grid amplification, parallel power combiner, quasi-optical beamforming.

\section{INTRODUCTION}

Increased propagation and material losses in combination with output power limitations of semiconductor devices represent a significant challenge for modern array antenna transmitters at mm-wave frequencies [1]. This challenge could be partly overcome by combining the power of multiple active devices on each antenna array element. However, conventional circuit-level power combining networks have inherently high insertion losses, which significantly increase with the number of active devices [2], [3], especially at higher frequencies. A possible solution toward more efficient high-power generation is the multi-channel transition in substrate integrated waveguide (SIW) technology employing spatial power combining [4]. This transition allows one to directly excite the $\mathrm{TE}_{10}$ SIW mode with high power. It avoids the use of a lossy on-chip circuit-level power combiner while enabling the direct integration of an antenna element with multiple power amplifiers (PAs) without additional interconnecting transitions. The advantages of the proposed solution become even more apparent for an increased number of input channels.

The challenge of the proposed solution is that mutual coupling effects between multiple channels have to be taken into account from the start of the design process. In [5] this transition has been optimized under the condition of a uniform excitation assuming identical PAs. However, realistic PAs are slightly different in their amplitude-phase characteristics due

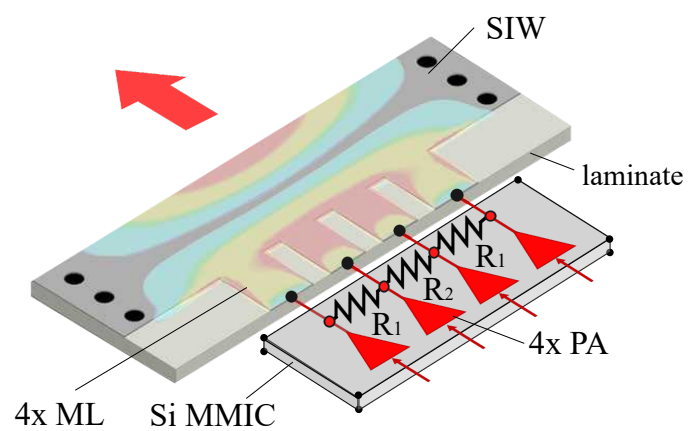

Fig. 1. The proposed spatial power combiner interfacing four on-chip amplifiers with isolation load resistors to a single substrate integrated waveguide.

to different thermal regimes, semiconductor process variations and fabrication tolerances.

This paper presents a more robust spatial power combiner (SPC) design relative to the one in [4] with enhanced performance figures in the presence of a non-uniform excitation. Fig. 1 illustrates this design solution, where three on-chip isolation load resistors are envisioned that are placed inbetween the four PA outputs.

\section{CONCEPT AND OPERATION PRINCIPLE}

In the original design [4] there are four input microstrip line (ML) ports with $50-\Omega$ reference impedance and a single matched SIW port as an output. It has been optimized to realize active impedance matching for the uniformly excited case. The active reflection coefficients at the transition input ports are defined as $\Gamma_{n}=\sum_{m=1}^{4} A_{m} S_{n m}$, where $S_{n m}$ is the $50-\Omega$ S-parameter from ML port $m$ to $n, A_{m}$ is a random variable representing the complex excitation coefficient of port $m$ simulating the effect of non-equal individual PAs. Generally, in order to reduce the effect of $\left\{A_{m}\right\}_{m=1}^{4}$ on the active reflections $\left\{\Gamma_{n}\right\}_{n=1}^{4}$, the magnitudes of $S_{n m}$ have to be minimized.

This minimization issue has been addressed by introducing isolation load resistors $\left(R_{1}, R_{2}\right)$ in-between the on-chip PA outputs (See Fig. 1). In the case of an ideal uniform excitation the voltage difference between the ML input ports will be zero implying that no current will flow into the isolation 
resistors and hence there is no power dissipation. For a non-uniform excitation, the differential-mode power will be largely dissipated in the resistors. This enhanced isolation between the channels results in smaller variation of the input port active reflection coefficients $\Gamma_{n}$. It is pointed out that, despite the power dissipation in the isolation resistors, the expected combined output power may still be higher than the scenario without resistors. This is owing to the behaviour of the individual PAs, which deliver less power if the output load impedance is mismatched [6].

\section{NUMERICAL VALIDATION RESULTS}

The optimal values of isolation load resistors have been found numerically by minimizing the coupling between the ML input ports while placing the resistors in-between those ports $^{1}$. The full-wave EM-simulated $5 \times 5$ scattering parameters of the SPC are shown in Fig. 2a. Due to the symmetry of the SIW structure, the isolation load resistors between symmetric ports must be equal. An isolation resistor interconnecting the edge ports ( 1 and 4 ) cannot be implemented in practice since it requires long interconnecting lines. Thus there are only two optimization parameters, i.e., $R_{1}$ and $R_{2}$, which have been found to be $108 \Omega$ and $150 \Omega$, respectively.

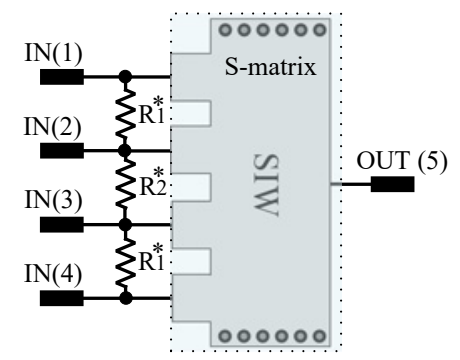

(a)

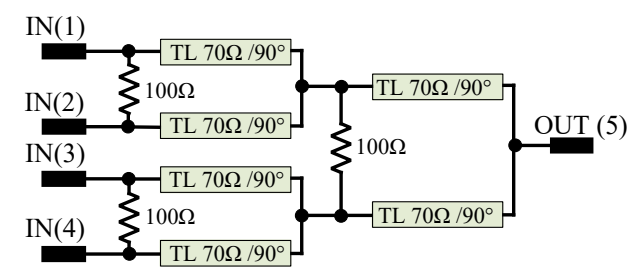

(b)

Fig. 2. Simulation models of the 4-way power combiners: (a) spatial power combiner $\mathrm{w} /$ and w/o isolation load resistors, (b) ideal Wilkinson power combiner.

For comparison, a 50- $\Omega$ Wilkinson power combiner built from ideal components was used as a reference [7]. It is formed by the connection of three $2: 1$ combiners each employing quarter-wave transmission lines (See Fig. 2b). The central operation frequency is $30 \mathrm{GHz}$.

\section{A. Scattering parameters}

The S-parameter simulations have been performed in Keysight's ADS software. Fig. 3a shows the simulated results

\footnotetext{
${ }^{1}$ The on-chip isolation load resistors are assumed ideal.
}

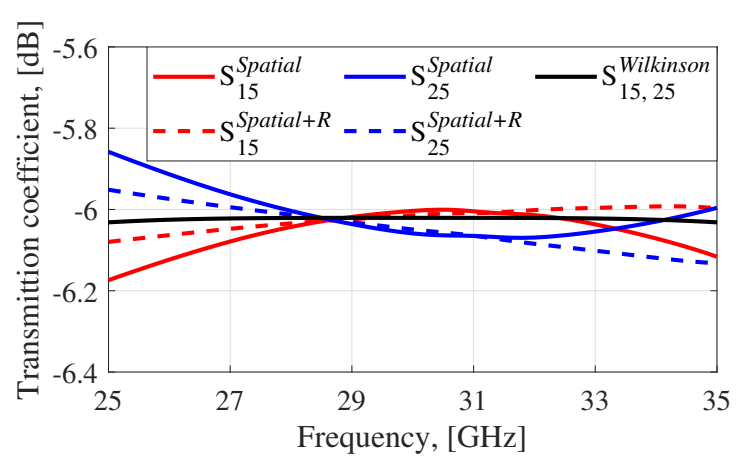

(a)

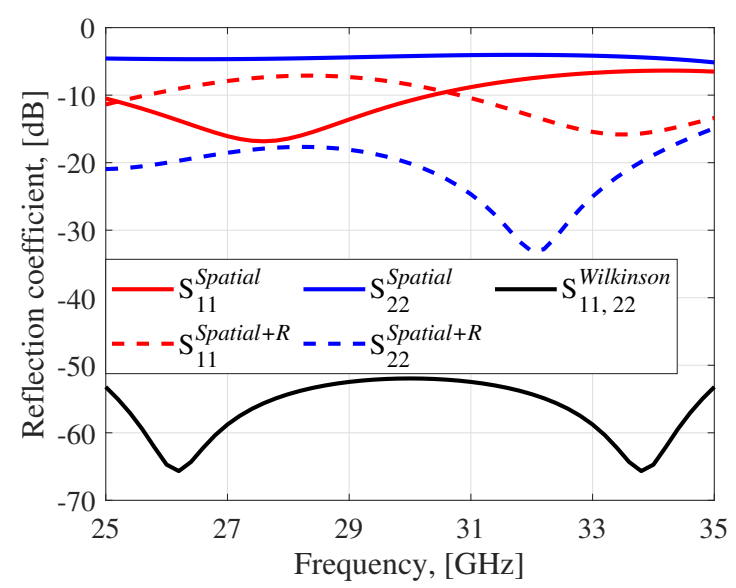

(b)

Fig. 3. (a) Simulated transmission, and (b) Simulated input port reflection coefficients coefficients of the spatial power combiner w/ and w/o isolation load resistors (dashed and solid line, resp.) and an ideal Wilkinson power combiner (black line). The results for symmetric ports are omitted.

of the SPC with and without the isolation load resistors (dashed and solid line, respectively) along with the Wilkinson power combiner case (black line). Since in the ideal 4:1 Wilkinson power combiner all input ports are identical, the transmission coefficients from each input port to the output port are equal to $-6 \mathrm{~dB}$ over a wide frequency range. This is different for the SPC, where the input ports are not identical due to the distributed $\mathrm{TE}_{10}$ mode structure. Therefore, using the isolation resistors allows to minimize the differences between the input channels and hence increase the efficiency of the PAs.

Fig. 3b shows the corresponding passive reflection coefficients. As one can see, the design solution with isolation resistors allows reducing reflections from port 2. The $S_{22}$ magnitude remains below $-15 \mathrm{~dB}$ over the $25-35 \mathrm{GHz}$ band, which is more than $13 \mathrm{~dB}$ lower in comparison to the case without resistors.

Fig. 4 shows the mutual coupling coefficients, where we observe much lower magnitude values in comparison to the original design with no isolation resistors. In particular, the $\left|S_{23}\right|$ and $\left|S_{13}\right|$ remain below $-15 \mathrm{~dB}$, and $\left|S_{12}\right|$ and $\left|S_{14}\right|$ remain below $-12 \mathrm{~dB}$ and $-7 \mathrm{~dB}$, respectively, over the 25- 


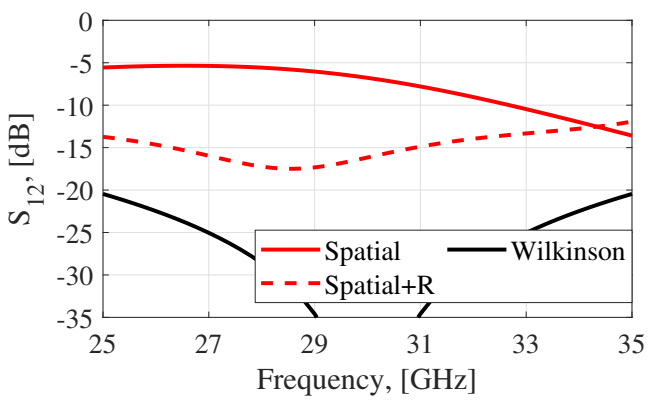

(a)

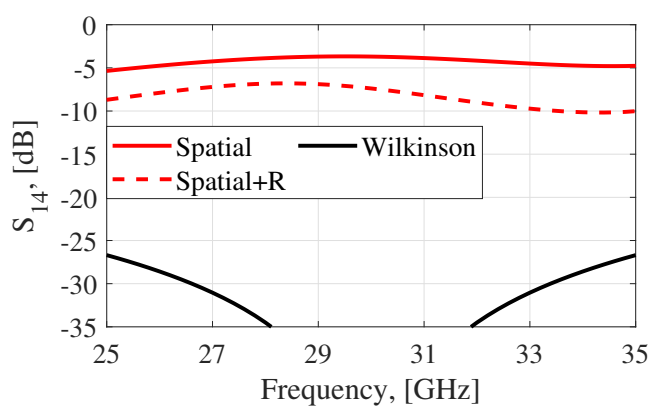

(c)

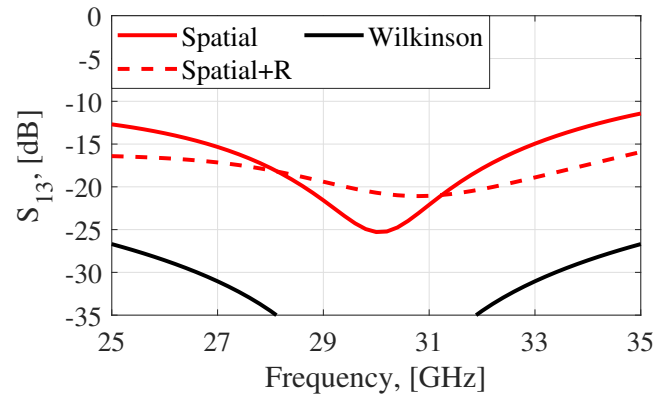

(b)

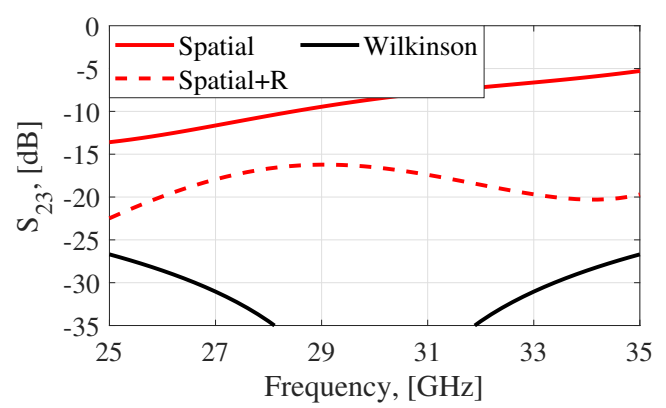

(d)

Fig. 4. Simulated mutual coupling between the input ports of the spatial power combiner w/ and w/o isolation load resistors (dashed and solid lines, respectively) and an ideal Wilkinson power combiner (black line). The results for symmetric ports are omitted.

$35 \mathrm{GHz}$ band. The relatively high level of $\left|S_{14}\right|$ could be reduced by introducing another resistor between the edge ports. The ideal Wilkinson combiner intrinsically has a good input matching and exhibits excellent isolation properties.

\section{B. Total performance in presence of PAs}

In order to evaluate the performance of the power combiners in conjunction with non-identical PAs, a simulation test-bench has been proposed (See Fig. 5). It has a single 50- $\Omega$ input port interfaced to an ideal 1:4 power splitter. The power splitter feeds an array of identical common-base PA-modules in SiGe HBT technology [8]. The power amplifiers are power-matched to the $50-\Omega$ load impedance.

In practice, phase variations of the PA gain dominate over amplitude drifts. This effect is emulated by four independent phase shifters with normally distributed phase values $\left\{\phi_{n}\right\}_{n=1}^{4}$. The normal distribution is defined by the mean value $\mu=0^{\circ}$ and standard deviation $\sigma=10^{\circ}$. The outputs of the PAs are interfaced to the matched 4:1 power combiners as discussed above.

The performance of such a joined active structure has been investigated in terms of PA metrics. Fig. 6 shows the gain, output power, and efficiency of a single PA at the 1$\mathrm{dB}$ compression point $(\mathrm{P} 1 \mathrm{~dB})$ over the $25-35 \mathrm{GHz}$ band. Ideally, the $\mathrm{P} 1 \mathrm{~dB}$ output power level after combining four PA channels should be $6 \mathrm{~dB}$ higher than that of a single PA, whereas the gain and efficiency values should remain the same. However, these performance targets are not feasible for realistic devices where phase variations are not negligible.

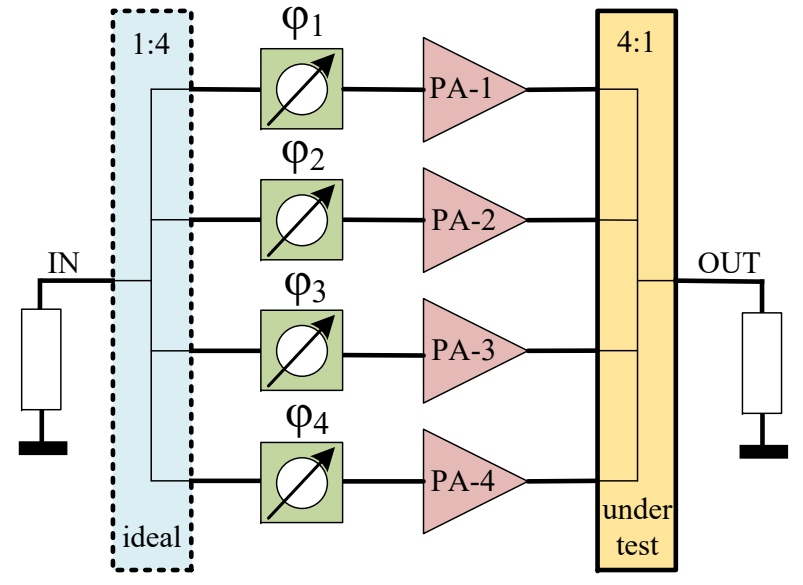

Fig. 5. Simulation test-bench for combined PA performance evaluation in the presence of normally distributed phase errors, used for the ideal Wilkinson power combiner and the spatial power combiners w/ and w/o isolation load resistors.

Fig. 7 shows the active reflection coefficients at the SPC input ports for the uniform excitation case. The level of reflections are below $-25 \mathrm{~dB}$ over the $25-35 \mathrm{GHz}$ band for all combiners, as expected.

Fig. 8 shows the simulated yield of the combined PAs as obtained with the test-bench in Fig. 5. The yield analysis is based on statistical variations of the introduced phase errors $\left\{\phi_{n}\right\}_{n=1}^{4}$. This is needed to determine how many possible 


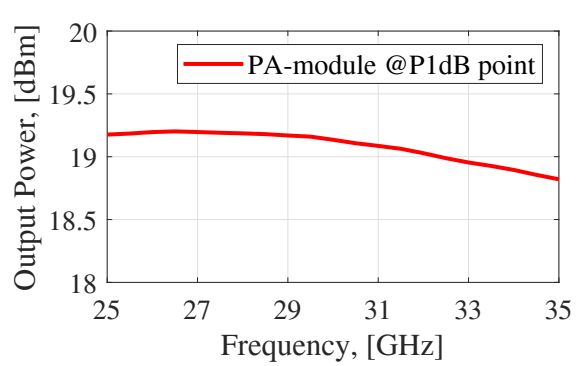

(a)

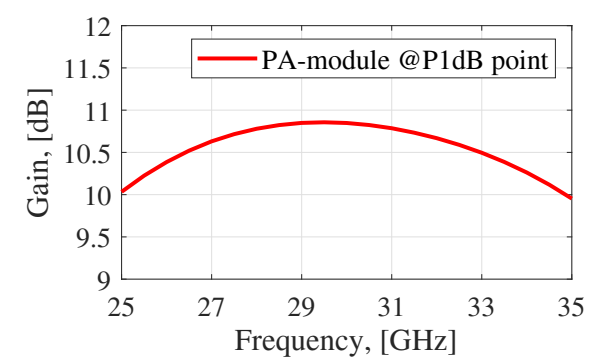

(b)

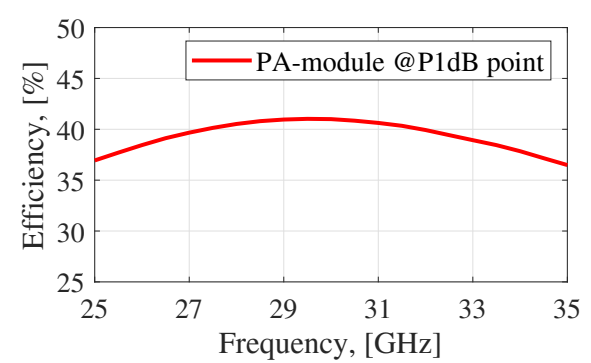

(c)

Fig. 6. Simulated performance of a single PA-module @P1dB compression point: (a) output power; (b) gain, and; (c) efficiency.

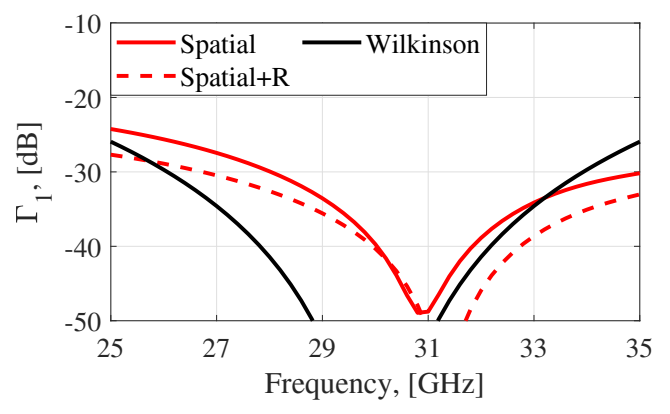

(a)

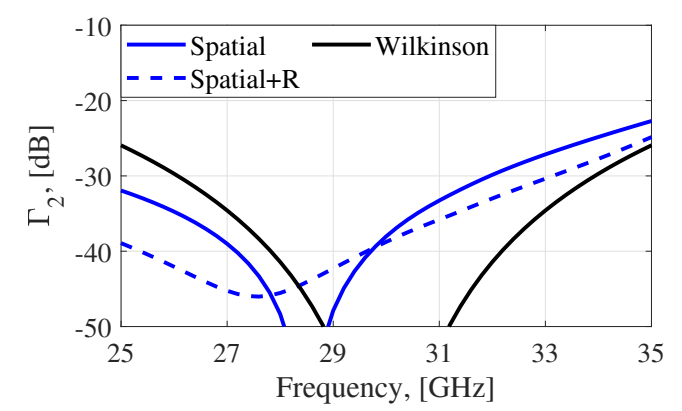

(b)

Fig. 7. Simulated input port active reflection coefficients (uniform excitation) of the spatial power combiner w/ and w/o isolation load resistors (dashed and solid lines, respectively) and the ideal Wilkinson power combiner (black). The results for symmetric ports are omitted.

phase combinations satisfy the following performance specifications which be met simultaneously:

- Output power ${ }^{2} \geq 24.5 \mathrm{dBm}$;

- Gain $^{2} \geq 10.5 \mathrm{~dB}$;

- Power efficiency ${ }^{2} \geq 35 \%$.

The colored region in Fig. 8 shows the operating bandwidth of a single PA-module, where the desired specifications could be potentially satisfied. It is seen that more than $90 \%$ and $80 \%$ of the realizations satisfy the desired specifications over the 29$31 \mathrm{GHz}$ and the $28-32 \mathrm{GHz}$ bands, respectively. It confirms that the proposed spatial power combiner with isolation load resistors is a good alternative to the conventional Wilkinson

\footnotetext{
2 Performance is at $1-\mathrm{dB}$ compression point.
}

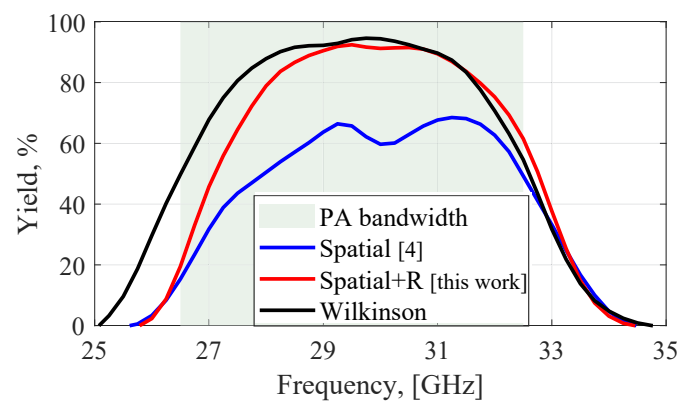

Fig. 8. Performance yield of the PAs in conjunction with the spatial power combiner w/ and w/o isolation load resistors (red and blue lines, respectively) and an ideal Wilkinson power combiner (black line) in the presence of normally distributed phase errors with $\sigma=10^{\circ}$. Positive performance yield if @P1dB, Gain $\geq 10.5 \mathrm{~dB}$, Power $\geq 24.5 \mathrm{~dB}$, Efficiency $\geq 35 \%$.

power combiner in terms of a low sensitivity to a non-uniform excitation.

\section{CONCLUSION}

A low-loss spatial power combiner with enhanced portisolation properties has been optimized and examined. It avoids the use of a lossy on-chip circuit-level power combiner while enabling the direct integration of an antenna element. The isolation load resistors allow reducing the coupling effects between PA channels, without increasing the losses in the case of a uniform excitation. The power combining performance in the presence of a non-uniform excitation caused by nonincidental PAs has been statistically analyzed. Simulations show that the proposed solution allows to significantly reduce the negative impact of a non-uniform excitation on the overall output power, gain, and power efficiency at the 1-dB compression point.

The concept is expected to play an important role in highpower MMICs, where both low insertion losses and optimal active device load matching are important requirements.

\section{ACKNOWLEDGMENT}

The work is a part of the Silicon-based Ka-band massive MIMO antenna systems for new telecommunication services (SILIKA) project, funded by the EU's Horizon 2020 under the Marie Skłodowska Curie grant agreement \#721732. 


\section{REFERENCES}

[1] J. Edstam, J. Hansryd, S. Carpenter et al. (2017, Feb.) Ericsson technology review: The new microwave backhaul frontier. [Online]. Available: https://www.ericsson.com/assets/local/publications/ericssontechnology-review/docs/2017/etr-beyond-100ghz.pdf

[2] M. P. DeLisio and R. A. York, "Quasi-optical and spatial power combining," IEEE Transactions on Microwave Theory and Techniques, vol. 50, no. 3, pp. 929-936, Mar 2002.

[3] J. Kim and G. M. Rebeiz, "Miniature four-way and two-way $24 \mathrm{GHz}$ wilkinson power dividers in $0.13 \mu \mathrm{m}$ CMOS," IEEE Microwave and Wireless Components Letters, vol. 17, no. 9, pp. 658-660, Sep. 2007.

[4] A. Roev, R. Maaskant, A. Höök, and M. Ivashina, "Wideband mm-wave transition between a coupled microstrip line array and SIW for highpower generation MMICs," IEEE Microwave and Wireless Components Letters, vol. 28, no. 10, pp. 867-869, Oct 2018.

[5] A. Roev, R. Maaskant, A. Hook, and M. Ivashina, "Efficient millimeterwave high power generation with spatial power-combined feeding element," in 2018 12th European Conference on Antennas and Propagation (EUCAP), Apr 2018, pp. 1-4.

[6] J. Staudinger, "Multiharmonic load termination effects on GaAs MESFET power amplifiers," Microwave J., vol. 39, no. 1, pp. 66-77, April 1996.

[7] E. J. Wilkinson, "An n-way hybrid power divider," IRE Transactions on Microwave Theory and Techniques, vol. 8, no. 1, pp. 116-118, January 1960.

[8] Y. Pei, "Silicon-based Ka-band reconfigurable phased-array transmitter," Ph.D. dissertation, Eindhoven University of Technology, Department of Electrical Engineering, Sept. 2016. 